\title{
An analytic expression for the electronic correlation term of the kinetic functional.
}

\author{
Luigi Delle Site* \\ Max-Planck-Institute for Polymer Research \\ Ackermannweg 10, D 55021 Mainz Germany.
}

\begin{abstract}
We propose an analytic formula for the non-local Fisher information functional, or electronic kinetic correlation term, appearing in the expression of the kinetic density functional. Such an explicit formula is constructed on the basis of well founded physical arguments and a rigorous mathematical prescription.
\end{abstract}

PACS numbers: 03.65. w, 71.10. w, 71.15.Mb

*Electronic address: dellsite@mpip-mainz.mpg.de 


\section{INTRODUCTION}

In a previous work we have treated the problem of a rigorous determination of the upper bound for the kinetic density functional of a system of electrons[1]. There, starting from the most general expression of a many-body wavefunction in polar form, a final formula was obtained where the kinetic functional consisted of two terms. The first, linked to the phase factor of the wavefunction, we have shown, can be bounded from above by a term proportional to the Thomas-Fermi one, the second corresponds to the Weizsacker term. A third term, corresponding to what is known as the non-local Fisher information functional, was neglected since, being quantitatively much smaller than the other terms [2], it was not relevant for the discussion carried on there. However, although quantitatively not relevant, such a term plays a fundamental role regarding important structural properties, such as the atomic shell structure, and it is strictly related to the electronic correlations implicit in the kinetic functional [3] (for this reason we have renamed it as "kinetic correlation term"). The difficulty in treating it is due to the fact that an explicit rigorous expression does not exists and to find a suitable one represents a challenging task [4]. To our knowledge, only few attempts have been made in this direction and they were restricted to highly simplified situations [5]. Clearly, for a detailed electronic description of condensed matter systems, this terms may play a key role. For this reason we focus the attention on such a problem and propose a general procedure, physically well founded and mathematically rigorous, to obtain an analytic expression. The practical importance of this problems stems from the fact that, as it is discussed in [1], there is a renewed interest towards energy functionals which are nowadays used in flexible quantum (based) computational methods, such as the linear-scaling real-space kinetic energy functional method, where the kinetic energy is calculated as a functional of the electron density. Such a method is also known as Orbital Free Density Functional (OFDFT) (see e.g. [6]). These modern methods allow in principle for the treatment of much larger systems and longer time scales than those based on standard Density Functional Theory in the Kohn-Sham formulation [7]. The crucial point of OFDFT, is that it is in principle exact, provided that the kinetic functional is rigorous. This means that the designing of rigorous kinetic density functionals would allow to explore the full power of the Hohenberg-Kohn theorem for extended systems. In the light of the discussion above, the search for a rigorous kinetic functional is not simply 
interesting for its own theoretical implications and understanding of fundamental aspect of quantum mechanics, but also for its large importance in practical calculations in condensed matter. The paper is organized as follows: the many-body wavefunction approach to obtain a $3 N$-dimensional functional (with $N$, the number of electrons), which was presented in our previous work, is reviewed and extended to the case where the electrons are explicitly interacting. Next the reduction to a 3-dimensional case for each term appearing in the $3 \mathrm{~N}$ dimensional expression is treated. While the term related to the wavefunction phase factor and the Coulomb interaction are known, the part related to the kinetic correlation is new and represent the core of this work.

\section{THE $N$-PARTICLE WAVEFUNCTION APPROACH FOR THE HAMILTO- NIAN PROBLEM}

Let us consider the many body Hamiltonian operator for a system of $N$ interacting electrons:

$$
H=-\frac{1}{2} \sum_{i=1}^{N} \nabla_{i}^{2}+\frac{1}{2} \sum_{j>i} \frac{1}{\left|\mathbf{r}_{i}-\mathbf{r}_{j}\right|}
$$

where for simplicity the Planck constant $\hbar$, the electron mass $m$ and the electron charge $e$ are taken to be 1 . Let us consider the most general form of the wavefunction solution of the stationary Schrödinger equation for an arbitrary state of energy $\epsilon$ :

$$
H \psi=\epsilon \psi
$$

Such a general form can be written as:

$$
\psi\left(\mathbf{r}_{1}, \ldots \ldots, \mathbf{r}_{N}\right)=e^{i S\left(\mathbf{r}_{1} \ldots \ldots, \mathbf{r}_{N}\right)} \Theta\left(\mathbf{r}_{1}, \ldots ., \mathbf{r}_{N}\right)
$$

with $S$ and $\Theta$ real functions in an $N$-dimensional space of volume $\Omega=\prod_{i=1}^{N} \omega_{i}=\omega^{N}$, with $\omega$ being the single particle volume. We also require $S$ to be symmetric and $\Theta$ to be antisymmetric with respect to any particle pair permutation. $\psi$ satisfies the normalization condition: $\int_{\Omega} \psi^{*} \psi d \mathbf{r}_{1} \ldots, d \mathbf{r}_{N}=1$. The spin variables are not explicitly considered, however, their effects will be properly taken into account and specifically mentioned when it will be required. Our aim is to use Eq, 2 and the form and properties of $\psi$ to derive an energy functional $E[\rho]=\int F[\rho(\mathbf{r})] d \mathbf{r}$ where $F[\rho(\mathbf{r})]$ is the energy density written as a functional of 
the one particle electron density, $\rho(\mathbf{r})$, defined as:

$$
\rho(\mathbf{r})=\rho\left(\mathbf{r}_{1}\right)=N \int_{\Omega_{N-1}} \psi^{*}\left(\mathbf{r}_{1}, \ldots, \mathbf{r}_{N}\right) \psi\left(\mathbf{r}_{1}, \ldots, \mathbf{r}_{N}\right) d \mathbf{r}_{2} \ldots \ldots d \mathbf{r}_{N}
$$

where $\Omega_{N-1}$ is the $N-1$-dimensional volume of $N-1$ particles, and $\int \rho(\mathbf{r}) d \mathbf{r}=N$; since the particles are indistinguishable, the index 1 can be exchanged with any other of the remaining $N-1$ particle index, for the rest of the work this formalism will be kept. $E[\rho]$ is the equivalent of $\epsilon$ in Eq, 2 written in terms of $\rho(\mathbf{r})$; thus we should obtain:

$$
E[\psi]=\int_{\Omega}\left(\psi^{*} H \psi\right) d \mathbf{r}_{1} \ldots d \mathbf{r}_{N}=\epsilon=E[\rho]=\int F[\rho(\mathbf{r})] d \mathbf{r} .
$$

To this aim we consider the $N$-particle energy density $\hat{F}_{\psi}=\psi^{*} H \psi$; the explicit expression is [1]:

$$
\begin{aligned}
\hat{F}_{\psi}= & \frac{1}{2} \sum_{i=1, N} \Theta^{2}\left(\mathbf{r}_{1}, . ., \mathbf{r}_{N}\right)\left|\nabla_{i} S\left(\mathbf{r}_{1}, . ., \mathbf{r}_{N}\right)\right|^{2}+\frac{1}{2} \sum_{i=1, N}\left|\nabla_{i} \Theta\left(\mathbf{r}_{1}, . ., \mathbf{r}_{N}\right)\right|^{2}+ \\
& +\frac{1}{2} \sum_{i=1}^{N} \sum_{j \neq i}^{1, N} \frac{\Theta^{2}\left(\mathbf{r}_{1}, . ., \mathbf{r}_{N}\right)}{\left|\mathbf{r}_{i}-\mathbf{r}_{j}\right|}-\frac{1}{4} \sum_{i=1, N} \nabla_{i}^{2} \Theta^{2}\left(\mathbf{r}_{1}, . ., \mathbf{r}_{N}\right) .
\end{aligned}
$$

Eq.6 is defined in a $3 N$-dimensional space; in order to derive $F[\rho(\mathbf{r})]$, we shall reduce the problem to a 3-dimensional space. Note that the last term on the r.h.s. vanishes after integration, thus it will not be relevant in this context. The first step to reduce the problem to a 3-dimensional space, is to redefine $\Theta^{2}\left(\mathbf{r}_{1}, \ldots . \mathbf{r}_{N}\right)$ in terms of a one particle probability density and an $(N-1)$-particle conditional probability density:

$$
\Theta^{2}\left(\mathbf{r}_{1}, \ldots, \mathbf{r}_{N}\right)=\frac{\rho\left(\mathbf{r}_{1}\right)}{N} f\left(\mathbf{r}_{2}, \ldots \ldots, \mathbf{r}_{N} / \mathbf{r}_{1}\right)
$$

where $=\frac{\rho\left(\mathbf{r}_{1}\right)}{N}$ is the one particle probability density and $f\left(\mathbf{r}_{2}, \ldots \ldots, \mathbf{r}_{N} / \mathbf{r}_{1}\right)$ is the $N-1$ electron conditional (w.r.t. $\mathbf{r}_{1}$ ) probability density, i.e. the probability density of finding an $N-1$ electron configuration, $C\left(\mathbf{r}_{2}, \ldots \ldots, \mathbf{r}_{N}\right)$, for a given fixed value of $\mathbf{r}_{1}$. The function $f$ satisfies the following property:

$$
\int_{\Omega_{N-1}} f\left(\mathbf{r}_{2}, \ldots \ldots ., \mathbf{r}_{N} / \mathbf{r}_{1}\right) d \mathbf{r}_{2} \ldots \ldots . d \mathbf{r}_{N}=1 \forall \mathbf{r}_{1}
$$

as before, due to the electron indistinguishability, the index 1 was chosen arbitrarily. Another equivalent factorization of $\Theta^{2}$, which it will be used for simplifying the electron-electron Coulomb term, consists of writing $\Theta^{2}$ as a product of a two particle probability density:

$$
\frac{\rho\left(\mathbf{r}_{1}, \mathbf{r}_{2}\right)}{N}=\int_{\Omega_{N-2}} \psi^{*}\left(\mathbf{r}_{1}, \mathbf{r}_{2}, \mathbf{r}_{3} \ldots, \mathbf{r}_{N}\right) \psi\left(\mathbf{r}_{1}, \mathbf{r}_{2}, \mathbf{r}_{3} \ldots, \mathbf{r}_{N}\right) d \mathbf{r}_{3} \ldots . . d \mathbf{r}_{N}
$$


equivalent to the diagonal element of the second-order spinless density matrix [7], and an $N-2$ particle conditional probability density:

$$
f\left(\mathbf{r}_{3}, \ldots \ldots, \mathbf{r}_{N} / \mathbf{r}_{1}, \mathbf{r}_{2}\right)
$$

Equivalently to the factorization shown before, in this case we have (see e.g. [8]):

$$
\int \rho\left(\mathbf{r}_{1}, \mathbf{r}_{2}\right) d \mathbf{r}_{1} d \mathbf{r}_{2}=N
$$

and:

$$
\int_{\Omega_{N-2}} f\left(\mathbf{r}_{3}, \ldots ., \mathbf{r}_{N} / \mathbf{r}_{1}, \mathbf{r}_{2}\right) d \mathbf{r}_{3} \ldots d \mathbf{r}_{N}=1 \forall \mathbf{r}_{1}, \mathbf{r}_{2}
$$

As a consequence of the definitions given above, the following identity is satisfied:

$$
\begin{aligned}
\Theta^{2}\left(\mathbf{r}_{1}, \ldots \mathbf{r}_{N}\right)= & \frac{\rho\left(\mathbf{r}_{1}\right)}{N} f\left(\mathbf{r}_{2}, . ., \mathbf{r}_{N} / \mathbf{r}_{1}\right)= \\
& =\frac{\rho\left(\mathbf{r}_{1}, \mathbf{r}_{2}\right)}{N} f\left(\mathbf{r}_{3}, . ., \mathbf{r}_{N} / \mathbf{r}_{1}, \mathbf{r}_{2}\right)
\end{aligned}
$$

Once again, since the particles are indistinguishable, the indices 1 and 2 for the one and two particle density were chosen arbitrarily and any other choice would be equivalent. Inserting Eq,7 and the r.h.s. of Eq.13 into Eq, $[$, using the properties of $f$ shown above, and remembering that the particles are indistinguishable, we obtain (for the kinetic terms see also [1, 10]):

$$
\begin{array}{r}
\int_{\Omega}\left(\psi^{*} H \psi\right) d \mathbf{r}_{1} \ldots d \mathbf{r}_{N}=\frac{1}{2} \int_{\Omega} \sum_{i=1}^{N} \Theta^{2}\left(\mathbf{r}_{1}, \ldots, \mathbf{r}_{N}\right)\left|\nabla_{i} S\left(\mathbf{r}_{1}, \ldots, \mathbf{r}_{N}\right)\right|^{2} d \mathbf{r}_{1} \ldots d \mathbf{r}_{N}+\frac{1}{8} \int_{\omega} \frac{\left|\nabla \rho\left(\mathbf{r}_{1}\right)\right|^{2}}{\rho\left(\mathbf{r}_{1}\right)} d \mathbf{r}_{1}+ \\
+\frac{1}{8} \int_{\omega} \rho\left(\mathbf{r}_{1}\right)\left[\int_{\Omega_{N-1}} \frac{\left.\mid \nabla_{\left.\mathbf{r}_{1} f\left(\mathbf{r}_{2}, \ldots, \mathbf{r}_{N} / \mathbf{r}_{1}\right)\right|^{2}}^{f\left(\mathbf{r}_{2}, \ldots, \mathbf{r}_{N} / \mathbf{r}_{1}\right)} d \mathbf{r}_{2} \ldots . d \mathbf{r}_{N}\right] d \mathbf{r}_{1}+}{\left.+\frac{(N-1)}{2} \int_{\omega} \int_{\omega} \frac{\rho\left(\mathbf{r}_{1}, \mathbf{r}_{2}\right)}{\left|\mathbf{r}_{1}-\mathbf{r}_{2}\right|} d \mathbf{r}_{1} d \mathbf{r}_{2} 14\right)}\right.
\end{array}
$$

The last term on the r.h.s. of Eq14 is obtained in the following way: first we use the property of particle indistinguishability and rewrite the Coulomb term in Eq.6 as :

$$
\frac{1}{2} \sum_{i=1}^{N} \sum_{j \neq i}^{1, N} \int_{\Omega} \frac{\left|\Theta\left(\mathbf{r}_{1}, \ldots, \mathbf{r}_{N}\right)\right|^{2}}{\left|\mathbf{r}_{i}-\mathbf{r}_{j}\right|} d \mathbf{r}_{1} \ldots d \mathbf{r}_{N}=\frac{N(N-1)}{2} \int_{\Omega} \frac{\left|\Theta\left(\mathbf{r}_{1}, \ldots, \mathbf{r}_{N}\right)\right|^{2}}{\left|\mathbf{r}_{1}-\mathbf{r}_{2}\right|} d \mathbf{r}_{1} \ldots d \mathbf{r}_{N}
$$

where the indices 1 and 2 are, as usual, chosen arbitrarily, then we use the decomposition of $\Theta^{2}$ as a product of the two particle density $\rho\left(\mathbf{r}_{1}, \mathbf{r}_{2}\right)$, and the two particle conditional 
probability density, $f\left(\mathbf{r}_{3}, \ldots, \mathbf{r}_{N} / \mathbf{r}_{1}, \mathbf{r}_{2}\right)$, and obtain:

$$
\begin{array}{r}
\frac{N(N-1)}{2} \int_{\Omega} \frac{\left|\Theta\left(\mathbf{r}_{1}, \ldots, \mathbf{r}_{N}\right)\right|^{2}}{\left|\mathbf{r}_{1}-\mathbf{r}_{2}\right|} d \mathbf{r}_{1} \ldots d \mathbf{r}_{N}=\frac{(N-1)}{2} \int_{\omega} \int_{\omega} \frac{\rho\left(\mathbf{r}_{1}, \mathbf{r}_{2}\right)}{\left|\mathbf{r}_{1}-\mathbf{r}_{2}\right|} d \mathbf{r}_{1} d \mathbf{r}_{2} \\
\int_{\Omega_{N-2}} f\left(\mathbf{r}_{3}, \ldots, \mathbf{r}_{N} / \mathbf{r}_{1}, \mathbf{r}_{2}\right) d \mathbf{r}_{3} \ldots . d \mathbf{r}_{N}
\end{array}
$$

Eq.16 can be simplified to a two particle expression, since

$$
\int_{\Omega_{N-2}} f\left(\mathbf{r}_{3}, \ldots \ldots, \mathbf{r}_{N} / \mathbf{r}_{1}, \mathbf{r}_{2}\right) d \mathbf{r}_{3} \ldots . d \mathbf{r}_{N}=1 \forall \mathbf{r}_{1}, \mathbf{r}_{2}
$$

so that we have:

$$
\frac{N(N-1)}{2} \int_{\Omega} \frac{\left|\Theta\left(\mathbf{r}_{1}, \ldots, \mathbf{r}_{N}\right)\right|^{2}}{\left|\mathbf{r}_{1}-\mathbf{r}_{2}\right|} d \mathbf{r}_{1} \ldots d \mathbf{r}_{N}=\frac{(N-1)}{2} \int_{\omega} \int_{\omega} \frac{\rho\left(\mathbf{r}_{1}, \mathbf{r}_{2}\right)}{\left|\mathbf{r}_{1}-\mathbf{r}_{2}\right|} d \mathbf{r}_{1} d \mathbf{r}_{2}
$$

\section{FROM THE $N$-PARTICLE TO THE ONE PARTICLE REPRESENTATION}

As one can see from Eq14 there are two kinetic terms which are written in an $N$-particle representation, namely, the phase factor term:

$$
\int_{\Omega} \frac{1}{2} \sum_{i=1}^{N} \Theta^{2}\left(\mathbf{r}_{1}, \ldots, \mathbf{r}_{N}\right)\left|\nabla_{i} S\left(\mathbf{r}_{1}, \ldots, \mathbf{r}_{N}\right)\right|^{2}
$$

and the correlation term also known as the Fisher non-local information functional [9, 10, 11, 12]:

$$
\frac{1}{8} \int_{\omega} \rho\left(\mathbf{r}_{1}\right) d \mathbf{r}_{1} \int_{\Omega_{N-1}} \frac{\left|\nabla_{\mathbf{r}_{1}} f\left(\mathbf{r}_{2}, \ldots ., \mathbf{r}_{N} / \mathbf{r}_{1}\right)\right|^{2}}{f\left(\mathbf{r}_{2}, \ldots ., \mathbf{r}_{N}\right)} d \mathbf{r}_{2} \ldots \ldots d \mathbf{r}_{N}
$$

and the aim of this work is to reduce them to a one-particle representation; the first term was already considered in a previous work [1] and the results will be reported here for completeness; the second term deserves a particular attention, since to our knowledge only very few attempts, restricted to specific and simple cases [5], were made to give an explicit formula; we will propose a rather general procedure to obtain an analytic expression. The third term, the electron-electron Coulomb interaction, which, as we have seen before, is a two particle term, and, generalizing the index 1 and 2, can be written as:

$$
E_{e e}=\frac{(N-1)}{2} \int_{\omega} \int_{\omega^{\prime}} \frac{\rho\left(\mathbf{r}, \mathbf{r}^{\prime}\right)}{\left|\mathbf{r}-\mathbf{r}^{\prime}\right|} d \mathbf{r} d \mathbf{r}^{\prime}
$$

it has been extensively treated in literature and the related results will play an important role in the procedure of reducing the $N$-particle expression of Eq20 to a single particle functional expression. 


\section{A. Reduction of $\int_{\Omega} \sum_{i=1, n} \Theta^{2}\left|\nabla_{i} S\right|^{2} d \mathbf{r}_{1} \ldots \mathbf{r}_{N}$ to a one particle representation}

In our previous work [1] we have shown that in the limit of a slowly varying density we can reasonably conjecture the following inequality:

$$
\left|\nabla_{i} S\right| \leq\left|\mathbf{P}_{F_{i}}\right|
$$

which means that the momentum of the $i$-th particle is less (or equal) to the Fermi momentum $P_{F_{i}}$ assigned to the $i$-th particle (i.e. the maximum value of the momentum an electron, under such conditions, can have). We have shown that Eq22 leads to the following inequality for the kinetic term related to the phase factor $S$ :

$$
\int_{\Omega} \sum_{i=1}^{N} \Theta^{2}\left(\mathbf{r}_{1}, \ldots, \mathbf{r}_{N}\right)\left|\nabla_{i} S\left(\mathbf{r}_{1}, \ldots, \mathbf{r}_{N}\right)\right|^{2} d \mathbf{r}_{1} \ldots d \mathbf{r}_{N} \leq C \int_{\omega} \rho^{\frac{5}{3}}(\mathbf{r}) d \mathbf{r} .
$$

Where $C=\frac{\left(3 \pi^{2}\right)^{2 / 3}}{2}$. We have also discussed the reasonability of our results beyond the slowly varying density regime and how our upper bound compares to others available in the literature. The effects of the spin are implicitly incorporated into the definition of $\mathbf{P}_{F_{i}}$, where the spin degeneracy enters as a scaling factor. On the other hand, any possible spin (or spatial) correlation effect implicit in $S$ can be neglected, because the energy assigned to each electron in the upper bound term is so large, or equivalently the overestimation of the energy is large enough, that any possible correlation energy can be reasonably neglected in Eq.23. In this case the term "reduction" means that the $3 N$-dimensional term is reduced to a 3-dimensional term, expressed through the quantity $\rho(\mathbf{r})$, which bounds the true expression from above. This is the first step in reducing $E[\psi]=\int_{\Omega} \psi^{*} H \psi d \mathbf{r}_{1} \ldots d \mathbf{r}_{N}$ to a reasonable density functional.

\section{B. The electron-electron interaction}

The electron-electron interaction energy $E_{e e}=\frac{(N-1)}{2} \int_{\omega} \int_{\omega^{\prime}} \frac{\rho\left(\mathbf{r}, \mathbf{r}^{\prime}\right)}{\left|\mathbf{r}-\mathbf{r}^{\prime}\right|} d \mathbf{r} d \mathbf{r}^{\prime}$, can be separated in two parts, the (classical) Hartree term:

$$
E_{H}=\int_{\omega} \int_{\omega^{\prime}} \frac{\rho(\mathbf{r}) \rho\left(\mathbf{r}^{\prime}\right)}{\left|\mathbf{r}-\mathbf{r}^{\prime}\right|} d \mathbf{r} d \mathbf{r}^{\prime}
$$

and the exchange and correlation term:

$$
E_{x c}=\int_{\omega} \rho(\mathbf{r}) \epsilon_{x c}(\mathbf{r}) d \mathbf{r}
$$


where $\epsilon_{x c}(\mathbf{r})$ is the exchange and correlation energy density (see e.g. [7]). In order to describe the nature of the exchange and correlation term in a simple and compact way, we refer to a series of seminal papers by Wigner and his coworker Seitz [13, 14]. In their work on the interaction of electrons in metals, they treat the case of uniformly distributed interacting electrons. They argue that the interaction energy of electrons with parallel spin is determined by the classical Coulomb interaction due to the space charge plus the exchange term. This latter, also known as Fermi correlation [15], is the consequence of the Pauli exclusion principle, and expresses the fact that two electrons with the same spin stay away from each other; such an effect is also called a Fermi hole. In contrast, the interaction of electrons with antiparallel spin contains the classical Coulomb term, plus the correlation term. The latter arises from the fact that electrons with antiparallel spin tend to condense in the same place but the electrostatic repulsion keeps them apart. We should also add that the exchange and correlation effects contained in the electron-electron term, are, by now, well understood. Although their satisfactory description is by far not easy achievable, there is a rich literature dealing with reasonable descriptions (numerical, analytical, semianalytical) of such a quantity (see e.g. articles in [16] and references therein). It is not the purpose of this work to propose anything new regarding this part; on the contrary, it is the availability of various analytical formulas for the exchange and correlation term which makes it possible to formally derive results on the kinetic correlation term.

\section{The non-local Fisher Information Functional or Kinetic Correlation term}

After having treated the kinetic term related to the wavefunction phase factor and having given a general description of the direct electron-electron interaction, we now come to the core of this work; to give a general prescription for finding an analytical functional form for the non local Fisher information functional or kinetic correlation term:

$$
\frac{1}{8} \int_{\omega} \rho(\mathbf{r}) I(\mathbf{r}) d \mathbf{r}=\frac{1}{8} \int_{\omega} \rho(\mathbf{r})\left[\int_{\Omega_{N-1}} \frac{\left|\nabla_{\mathbf{r}} f\left(\mathbf{r}_{2}, \ldots . \mathbf{r}_{N} / \mathbf{r}\right)\right|^{2}}{f\left(\mathbf{r}_{2}, \ldots . \mathbf{r}_{N} / \mathbf{r}\right)} d \mathbf{r}_{2} \ldots d \mathbf{r}_{N}\right] d \mathbf{r} .
$$

The main point is to give physically as well as mathematically well founded conditions which, when satisfied, lead to a valid expression for $I(\mathbf{r})$. This term is qualitatively very important since it is responsible of the atomic shell structure [3, 5], thus to have a valid description

of it automatically means to have an accurate description of the electronic structure. Inter- 
estingly, Ludeña and Karasiev[3] intuitively link this term to the exchange and correlation energy, although a prescription for how to find this link is not given. We start from the same intuitive idea and develop it further. The essence of the idea developed here is that a gas of electrons, as long as these are not related by an explicit interaction, does not display correlation effects, except those of the basic Fermi distribution, or occupation of levels, for non interacting electrons. However, when a direct electron-electron interaction is switched on, then the electrons start to feel the presence of all the others in a non trivial way. This means that their spatial distribution is heavily governed by the fact that they try to optimize such correlations (interactions) in a way that the optimal spatial arrangement fulfills the minimum energy requirements. For this reason the key quantity determining the many particle electron distribution is the electron-electron interaction:

$$
E_{e e}\left(\mathbf{r}, \mathbf{r}^{\prime}\right)=\frac{\rho\left(\mathbf{r}, \mathbf{r}^{\prime}\right)}{\left|\mathbf{r}-\mathbf{r}^{\prime}\right|}
$$

The expression of Eq27 is a two-particle function, averaged over the rest of $N-2$ particles, where the effects of these latter on the electrons at $\mathbf{r}$ and $\mathbf{r}^{\prime}$ is implicitly described. As we have seen before, although we do not have an exact expression for $E_{e e}\left(\mathbf{r}, \mathbf{r}^{\prime}\right)$, nevertheless several valid approximations are given in an analytic form. Conversely, very little can be found in the literature for $I(\mathbf{r})$ due to the difficulty in writing an explicit expression for $f\left(\mathbf{r}_{2}, \ldots, \mathbf{r}_{N} / \mathbf{r}\right)$ [4]. For this reason, since we cannot write directly $f\left(\mathbf{r}_{2}, \ldots, \mathbf{r}_{N} / \mathbf{r}\right)$ in an explicit way, we can think of transforming $f\left(\mathbf{r}_{2}, \ldots, \mathbf{r}_{N} / \mathbf{r}\right)$ into some function of $E_{e e}\left(\mathbf{r}, \mathbf{r}^{\prime}\right)$; this approach, although indirect, can lead to a physically well-founded analytic expression. As said before, $f\left(\mathbf{r}_{2}, \ldots, \mathbf{r}_{N} / \mathbf{r}\right)$ is the probability density of finding $N-1$ particles in a certain configuration once one particle $\mathbf{r}$ is fixed; it is reasonable to think that it must be linked to the electron-electron interaction of Eq.27. For the reasons given above we propose the following conjecture:

$$
f\left(\mathbf{r}_{2}, \ldots, \mathbf{r}_{N} / \mathbf{r}\right)=\Pi_{i=2}^{N} h_{i}\left(E_{e e}\left(\mathbf{r}, \mathbf{r}_{i}\right)\right)
$$

where $h_{i}\left(E_{e e}\left(\mathbf{r}, \mathbf{r}_{i}\right)\right)$ must satisfies the following conditions:

(a) $\int_{\omega} h_{i}\left(E_{e e}\left(\mathbf{r}, \mathbf{r}_{i}\right)\right) d \mathbf{r}_{i}=1 ; \forall \mathbf{r}$

(b) $\quad h_{i}\left(E_{e e}(\mathbf{r}, \mathbf{r})\right)=0$

(c) $\Pi_{i=2}^{N} h_{i}\left(E_{e e}\left(\mathbf{r}, \mathbf{r}_{i}\right)\right) \leq 1 ; \forall \mathbf{r}, \mathbf{r}_{i}$. 
Condition (a) comes directly from Eq, $[$, condition $(b)$ is the direct consequence of the antisymmetry of $\psi$, while the last condition expresses the fact that $f\left(\mathbf{r}_{2}, \ldots, \mathbf{r}_{N} / \mathbf{r}\right)$ is a probability density, thus cannot be larger than one. The function $\Pi_{i=2}^{N} h_{i}\left(E_{e e}\left(\mathbf{r}, \mathbf{r}_{i}\right)\right)$ in this context should be interpreted as the probability density which expresses how the $\mathbf{r}_{i}$ particles distribute themselves w.r.t. the r-particle (fixed), while experiencing each other (except $\mathbf{r}$ ) in an average way implicitly contained in $E_{e e}\left(\mathbf{r}, \mathbf{r}^{\prime}\right)$ through $\rho(\mathbf{r})$. The conditions in Eq 29 are general, and can be used to design possible functional forms for $I(\mathbf{r})$. In the next section we propose some functional forms and discuss the consequences.

\section{The exponential form}

In this section we will treat the case when the function $h_{i}=h_{i}\left(E_{e e}\left(\mathbf{r}, \mathbf{r}_{i}\right)\right)$ has the form of an exponential. By this ansatz and its related mathematical prescription we will gain some interesting insights into, possibly, physical effects related to the kinetic correlation term we are treating. The functional form we propose for $f$ has the following expression:

$$
\Pi_{i=2}^{N} h_{i}\left(E_{e e}\left(\mathbf{r}, \mathbf{r}_{i}\right)\right)=\Pi_{i=2}^{N} e^{(N-1) \bar{E}(\mathbf{r})} e^{-E_{e e}\left(\mathbf{r}, \mathbf{r}_{i}\right)}
$$

where

$$
e^{-\bar{E}(\mathbf{r})}=\int_{\omega} e^{-E_{e e}\left(\mathbf{r}, \mathbf{r}_{i}\right)} d \mathbf{r}_{i}
$$

This function naturally satisfies the requirement $(a)$ and $(c)$ of Eq. 29 , in fact

$$
e^{\bar{E}(\mathbf{r})} \times \int_{\omega} e^{-E_{e e}\left(\mathbf{r}, \mathbf{r}_{i}\right)} d \mathbf{r}_{i}=1 ; \forall \mathbf{r}, i
$$

and as a consequence, because of the particles' indistinguishability, we have:

$$
\int_{\Omega_{N-1}} \Pi_{i=2}^{N} e^{(N-1) \bar{E}(\mathbf{r})} e^{-E_{e e}\left(\mathbf{r}, \mathbf{r}_{i}\right)} d \mathbf{r}_{2} \ldots d \mathbf{r}_{N}=1 ; \forall \mathbf{r}
$$

Condition $(c)$ is satisfied, since $e^{\bar{E}(\mathbf{r})}$ is the automatic normalization factor, and thus:

$$
\frac{e^{-E_{e e}\left(\mathbf{r}, \mathbf{r}_{i}\right)}}{e^{-\bar{E}(\mathbf{r})}} \leq 1 ; \forall \mathbf{r}, \mathbf{r}_{i}
$$

and again, this can be straightforwardly generalized to the complete $\prod_{i=2}^{N} h_{i}\left(E_{e e}\left(\mathbf{r}, \mathbf{r}_{i}\right)\right)$ function. We will come back to condition $(b)$ of Eq.29 later on, for the moment let us justify why the exponential form should be physically sound. The starting point is that the 
electron-electron interaction is a positive definite function, $E_{e e}\left(\mathbf{r}, \mathbf{r}^{\prime}\right) \geq 0 ; \forall \mathbf{r}, \mathbf{r}^{\prime}$. The physical principle we use is that the electrons try to distribute themselves in a way that their spatial arrangement is consistent with the optimal electron-electron total interaction energy. In this context the conditional probability density, $f\left(\mathbf{r}_{2}, \ldots \mathbf{r}_{N} / \mathbf{r}\right)=\prod_{i=2}^{N} \frac{e^{-E_{e e}\left(\mathbf{r}, \mathbf{r}_{i}\right)}}{e^{-(N-1) \bar{E}(\mathbf{r})}}$ expresses the fact that for a configuration of high interaction the probability is low because electrons tend to avoid each other; on the other hand, for a lower interaction energy the probability is higher, because the electrons can distribute in a more optimal way. So far, on the basis of the physical intuition and mathematical requirements, our ansatz seems rather reasonable; however in this form, $f\left(\mathbf{r}_{2}, \ldots \mathbf{r}_{N} / \mathbf{r}\right)$ does not satisfy a major symmetry requirement, namely condition $(b)$ of Eq,29. In fact $\Theta\left(\mathbf{r}_{1}, . . \mathbf{r}_{N}\right)$ being antisymmetric under any particle pair permutation, it would require that $\Theta^{2}\left(\mathbf{r}, \mathbf{r}_{2}, \ldots \mathbf{r}, \ldots \mathbf{r}_{N}\right)=0$. We should remember that $\Theta^{2}\left(\mathbf{r}, \mathbf{r}_{2}, \ldots \mathbf{r}, \ldots \mathbf{r}_{N}\right)=\rho(\mathbf{r}) f\left(\mathbf{r}_{2}, \ldots \mathbf{r}_{N} / \mathbf{r}\right)$, which implies that $f\left(\mathbf{r}_{2}, ., \mathbf{r}, \ldots, \mathbf{r}_{N} / \mathbf{r}\right)=0$; this is impossible in the form we have expressed $f\left(\mathbf{r}_{2}, \ldots \mathbf{r}_{N} / \mathbf{r}\right)$. To understand this point we have to look at the explicit expression of $E_{e e}$ :

$$
E_{e e}\left(\mathbf{r}, \mathbf{r}^{\prime}\right)=\frac{\rho\left(\mathbf{r}, \mathbf{r}^{\prime}\right)}{\left|\mathbf{r}-\mathbf{r}^{\prime}\right|}
$$

thus:

$$
\lim _{\mathbf{r} \rightarrow \mathbf{r}^{\prime}} E_{e e}\left(\mathbf{r}, \mathbf{r}^{\prime}\right)=0
$$

i.e. the probability of finding two electrons in the same position is zero, and thus the related interaction is zero too. This can be also seen, for example, in Ref.[15], where $E_{e e}\left(\mathbf{r}, \mathbf{r}^{\prime}\right)$ is explicitly written in term of classical Coulomb interaction plus the exchange and correlation term; for $\mathbf{r} \rightarrow \mathbf{r}^{\prime}$ the exchange and correlation part becomes equal to minus the classical Coulomb term, and thus they cancel each other. For our expression of $f$, instead, we must require $E_{e e}\left(\mathbf{r}, \mathbf{r}^{\prime}\right) \rightarrow \infty$ for $\mathbf{r}^{\prime} \rightarrow \mathbf{r}$ so that $f\left(\mathbf{r}_{2}, ., \mathbf{r}, . ., \mathbf{r}_{N} / \mathbf{r}\right)=0$, however this is not possible because $E_{e e}(\mathbf{r}, \mathbf{r})=0$ thus we cannot use $E_{e e}\left(\mathbf{r}, \mathbf{r}^{\prime}\right)$ as a central quantity. There is also another necessary condition which must be satisfied by $f\left(\mathbf{r}_{2}, . ., \mathbf{r}_{N} / \mathbf{r}\right)$, and it involves directly the kinetic correlation term; in fact, this latter must be finite whenever any one of the $\mathbf{r}_{i}$ electrons approaches the r-electron; i.e.

$$
\frac{\left|\nabla_{\mathbf{r}} f\left(\mathbf{r}_{2}, . ., \ldots \mathbf{r}, \ldots, \mathbf{r}_{N} / \mathbf{r}\right)\right|^{2}}{f\left(\mathbf{r}_{2}, . ., \mathbf{r}, \ldots, \mathbf{r}_{N} / \mathbf{r}\right)}=\text { finite }
$$

where finite is in general either zero or a finite positive number or even a non diverging function of the other variables than $\mathbf{r}$. Eq.37 implies that the numerator should go to zero 
with the same order of, or faster than, the denominator. This mathematical requirement together with condition (b) of Eq,29 suggest that instead of the full electron-electron interaction, $E_{e e}\left(\mathbf{r}, \mathbf{r}^{\prime}\right)$, we should use only the classical Coulomb term, also known as the Hartree term, $E_{H}\left(\mathbf{r}, \mathbf{r}^{\prime}\right)=\frac{\rho(\mathbf{r}) \rho\left(\mathbf{r}^{\prime}\right)}{\left|\mathbf{r}-\mathbf{r}^{\prime}\right|}$. It can be easily verified that it satisfies the conditions $(a),(b)$, (c) of Eq,29, and leads to a finite value (zero) for the kinetic correlation term in the limit of $\mathbf{r}_{i} \rightarrow \mathbf{r}$. In fact in this case one has:

$$
\frac{\left|\nabla_{\mathbf{r}} f\left(\mathbf{r}_{2}, . ., \mathbf{r}_{N} / \mathbf{r}\right)\right|^{2}}{f\left(\mathbf{r}_{2}, . ., \mathbf{r}_{N} / \mathbf{r}\right)}=\frac{\left|\nabla_{\mathbf{r}}\left[\Pi_{i=2}^{N} e^{(N-1) \bar{E}(\mathbf{r})} e^{-E_{H}\left(\mathbf{r}, \mathbf{r}_{i}\right)}\right]\right|^{2}}{\left[\Pi_{i=2}^{N} e^{(N-1) \bar{E}(\mathbf{r})} e^{-E_{H}\left(\mathbf{r}, \mathbf{r}_{i}\right)}\right]}
$$

The r.h.s. of Eq 38 becomes:

$$
e^{(N-1) \bar{E}(\mathbf{r})} \Pi_{i=2}^{N} e^{-E\left(\mathbf{r}, \mathbf{r}_{i}\right)} \cdot\left|(N-1) \nabla_{\mathbf{r}} \bar{E}(\mathbf{r})-\sum_{i=2}^{N} \nabla_{\mathbf{r}} E_{H}\left(\mathbf{r}, \mathbf{r}_{i}\right)\right|^{2}
$$

and thus we have:

$$
\lim _{\mathbf{r}_{i} \rightarrow \mathbf{r}} e^{(N-1) \bar{E}(\mathbf{r})} \Pi_{i=2}^{N} e^{-E\left(\mathbf{r}, \mathbf{r}_{i}\right)} \cdot\left|(N-1) \nabla_{\mathbf{r}} \bar{E}(\mathbf{r})-\sum_{i=2}^{N} \nabla_{\mathbf{r}} E_{H}\left(\mathbf{r}, \mathbf{r}_{i}\right)\right|^{2}=0 ; \forall \mathbf{r}_{i}
$$

The limit in Eq40 is zero because while $\left|(N-1) \nabla_{\mathbf{r}} \bar{E}(\mathbf{r})-\sum_{i=2}^{N} \nabla_{\mathbf{r}} E_{H}\left(\mathbf{r}, \mathbf{r}_{i}\right)\right|^{2}$ diverges for $\mathbf{r}_{i} \rightarrow \mathbf{r}$, in the worst case, as $\left|\mathbf{r}-\mathbf{r}_{i}\right|^{-n}$ with $n$ a finite positive integer, the exponential $e^{-E_{H}\left(\mathbf{r}, \mathbf{r}_{i}\right)}$ tends to zero in a much faster way than the divergent term. The fact that $I(\mathbf{r})$, written using the exponential form of $f$, does not diverge for $\mathbf{r}_{i} \rightarrow \mathbf{r}$, together with the other mathematical properties discussed before, show that indeed the exponential ansatz is mathematically and physically sound, thus we can proceed to the core of this work, that is to write an explicit formula for $I(\mathbf{r})$. From Eq 38 and Eq 39 we obtain:

$$
\begin{array}{r}
I(\mathbf{r})=(N-1)^{2}\left|\nabla_{\mathbf{r}} \bar{E}(\mathbf{r})\right|^{2} \int_{\omega} e^{(N-1) \bar{E}(\mathbf{r})} \Pi_{i=2}^{N} e^{-E_{H}\left(\mathbf{r}, \mathbf{r}_{i}\right)} d \mathbf{r}_{2} \ldots d \mathbf{r}_{N}- \\
-2(N-1) \sum_{i=2}^{N} \int_{\omega}\left[\int_{\Omega_{N-2}} e^{(N-2) \bar{E}(\mathbf{r})} \Pi_{l \neq i} e^{-E_{H}\left(\mathbf{r}, \mathbf{r}_{l}\right)} d \mathbf{r}_{l}\right] e^{\bar{E}(\mathbf{r})-E_{H}\left(\mathbf{r}, \mathbf{r}_{i}\right)}\left[\nabla_{\mathbf{r}} \bar{E}(\mathbf{r}) \cdot \nabla_{\mathbf{r}} E_{H}\left(\mathbf{r}, \mathbf{r}_{i}\right)\right] d \mathbf{r}_{i}+ \\
+\sum_{i=2}^{N} \int_{\omega}\left[\int_{\Omega_{N-2}} e^{(N-2) \bar{E}(\mathbf{r})} \Pi_{l \neq i} e^{-E_{H}\left(\mathbf{r}, \mathbf{r}_{l}\right)} d \mathbf{r}_{l}\right] e^{\bar{E}(\mathbf{r})-E_{H}\left(\mathbf{r}, \mathbf{r}_{i}\right)}\left|\nabla_{\mathbf{r}} E_{H}\left(\mathbf{r}, \mathbf{r}_{i}\right)\right|^{2} d \mathbf{r}_{i}+ \\
+\sum_{i} \sum_{j \neq i} \int_{\omega} \int_{\omega}\left[\int_{\Omega_{N-3}} e^{(N-3) \bar{E}(\mathbf{r})} \Pi_{l \neq i ; l \neq j} e^{-E\left(\mathbf{r}, \mathbf{r}_{l}\right)} d \mathbf{r}_{l}\right] e^{\bar{E}(\mathbf{r})-E\left(\mathbf{r}, \mathbf{r}_{i}\right)} e^{\bar{E}(\mathbf{r})-E\left(\mathbf{r}, \mathbf{r}_{j}\right)} \times \\
\times\left|\nabla_{\mathbf{r}} E\left(\mathbf{r}, \mathbf{r}_{i}\right) \cdot \nabla_{\mathbf{r}} E\left(\mathbf{r}, \mathbf{r}_{j}\right)\right| d \mathbf{r}_{i} d \mathbf{r}_{j}(41)
\end{array}
$$

Using the indistinguishability, taking into account that all the expressions of the form $\int e^{\bar{E}(\mathbf{r})-E\left(\mathbf{r}, \mathbf{r}_{l}\right)} d \mathbf{r}_{l}=1 ; \forall \mathbf{r}$ and factorizing all the $\mathbf{r}_{l}$ 's that do not contain the variable $\mathbf{r}_{i}$ present 
in $\nabla_{\mathbf{r}} E\left(\mathbf{r}, \mathbf{r}_{i}\right)$, we obtain:

$$
\begin{array}{r}
I(\mathbf{r})=(N-1)^{2}|\bar{E}(\mathbf{r})|^{2}-2(N-1)^{2} \int_{\omega} e^{\bar{E}(\mathbf{r})-E\left(\mathbf{r}, \mathbf{r}^{\prime}\right)} \nabla_{\mathbf{r}} \bar{E}(\mathbf{r}) \cdot \nabla_{\mathbf{r}} E\left(\mathbf{r}, \mathbf{r}^{\prime}\right) d \mathbf{r}^{\prime}+ \\
+(N-1) \int_{\omega} e^{\bar{E}(\mathbf{r})-E\left(\mathbf{r}, \mathbf{r}^{\prime}\right)}\left|\nabla_{\mathbf{r}} E\left(\mathbf{r}, \mathbf{r}^{\prime}\right)\right|^{2} d \mathbf{r}^{\prime}+ \\
+(N-1)(N-2) \int_{\omega} \int_{\omega} e^{\bar{E}(\mathbf{r})-E\left(\mathbf{r}, \mathbf{r}^{\prime}\right)} e^{\bar{E}(\mathbf{r})-E\left(\mathbf{r}, \mathbf{r}^{\prime \prime}\right)} \nabla_{\mathbf{r}} E\left(\mathbf{r}, \mathbf{r}^{\prime}\right) \cdot \nabla_{\mathbf{r}} E\left(\mathbf{r}, \mathbf{r}^{\prime \prime}\right) d \mathbf{r}^{\prime} d \mathbf{r}^{\prime \prime} .
\end{array}
$$

The last term on the r.h.s. of Eq42,characterized by a sort of double independent non locality, $\left(\mathbf{r}^{\prime}\right.$ and $\left.\mathbf{r}^{\prime \prime}\right)$, can be reduced to a simple non locality since:

$$
\nabla_{\mathbf{r}} E\left(\mathbf{r}, \mathbf{r}^{\prime}\right) \cdot \nabla_{\mathbf{r}} E\left(\mathbf{r}, \mathbf{r}^{\prime \prime}\right)=\partial_{x} E\left(\mathbf{r}, \mathbf{r}^{\prime}\right) \partial_{x} E\left(\mathbf{r}, \mathbf{r}^{\prime \prime}\right)+\partial_{y} E\left(\mathbf{r}, \mathbf{r}^{\prime}\right) \partial_{y} E\left(\mathbf{r}, \mathbf{r}^{\prime \prime}\right)+\partial_{z} E\left(\mathbf{r}, \mathbf{r}^{\prime}\right) \partial_{z} E\left(\mathbf{r}, \mathbf{r}^{\prime \prime}\right)
$$

thus:

$$
\begin{aligned}
& (N-1)(N-2) \int_{\omega} \int_{\omega} e^{\bar{E}(\mathbf{r})-E\left(\mathbf{r}, \mathbf{r}^{\prime}\right)} e^{\bar{E}(\mathbf{r})-E\left(\mathbf{r}, \mathbf{r}^{\prime \prime}\right)} \nabla_{\mathbf{r}} E\left(\mathbf{r}, \mathbf{r}^{\prime}\right) \cdot \nabla_{\mathbf{r}} E\left(\mathbf{r}, \mathbf{r}^{\prime \prime}\right) d \mathbf{r}^{\prime} d \mathbf{r}^{\prime \prime}= \\
& =(N-1)(N-2) e^{2 \bar{E}(\mathbf{r})} \sum_{k=1,3} \int_{\omega} e^{E\left(\mathbf{r}, \mathbf{r}^{\prime}\right)} \partial_{x_{k}} E\left(\mathbf{r}, \mathbf{r}^{\prime}\right) d \mathbf{r}^{\prime} \int_{\omega} e^{E\left(\mathbf{r}, \mathbf{r}^{\prime \prime}\right)} \partial_{x_{k}} E\left(\mathbf{r}, \mathbf{r}^{\prime \prime}\right) d \mathbf{r}^{\prime \prime}
\end{aligned}
$$

where $x_{1}=x ; x_{2}=y ; x_{3}=z$, and from Eq44, since $\partial_{x_{k}} E\left(\mathbf{r}, \mathbf{r}^{\prime}\right)$ and $\partial_{x_{k}} E\left(\mathbf{r}, \mathbf{r}^{\prime \prime}\right)$ are independent from each other, one obtains:

$e^{2 \bar{E}(\mathbf{r})} \sum_{k=1,3} \int_{\omega} e^{E\left(\mathbf{r}, \mathbf{r}^{\prime}\right)} \partial_{x_{k}} E\left(\mathbf{r}, \mathbf{r}^{\prime}\right) d \mathbf{r}^{\prime} \int_{\omega} e^{E\left(\mathbf{r}, \mathbf{r}^{\prime \prime}\right)} \partial_{x_{k}} E\left(\mathbf{r}, \mathbf{r}^{\prime \prime}\right) d \mathbf{r}^{\prime \prime}=e^{2 \bar{E}(\mathbf{r})} \sum_{k=1,3}\left[\int_{\omega} e^{E\left(\mathbf{r}, \mathbf{r}^{\prime}\right)} \partial_{x_{k}} E\left(\mathbf{r}, \mathbf{r}^{\prime}\right) d \mathbf{r}^{\prime}\right]^{2}$.

Inserting $\mathrm{Eq} 45$ in $\mathrm{Eq} 42$, we obtain an analytic expression for $I(\mathbf{r})$, since all the terms involved in Eq42 and Eq45 are known explicitly:

$$
E_{H}\left(\mathbf{r}, \mathbf{r}^{\prime}\right)=\frac{\rho(\mathbf{r}) \rho\left(\mathbf{r}^{\prime}\right)}{\left|\mathbf{r}-\mathbf{r}^{\prime}\right|}
$$

and;

$$
\bar{E}(\mathbf{r})=-\log \left[\int_{\omega} e^{-E_{H}\left(\mathbf{r}, \mathbf{r}^{\prime}\right)} d \mathbf{r}^{\prime}\right]
$$

The physical interpretation of the results obtained is the following, the non local Fisher information functional or kinetic correlation functional, can be well described by the classical Coulomb interaction between the electrons. Such a conclusion is based on the fact that the exponential form is physically "reasonable", however the related mathematical prescriptions do exclude that the exchange and correlation interaction plays a role in this case. Rather, it leads to the conclusion that the classical Coulomb interaction naturally dominates the kinetic correlation effects since it perfectly matches the physical arguments and the mathematical requirements. 


\section{Discussion and Conclusions}

The proposed exponential form for the conditional probability has been obtained on the basis of well founded physical arguments and rigorous mathematical prescriptions. Interestingly, starting from a rather different point of view and aiming at a different goal, Ceperly [17] and Jastrow [18], choose, on the basis of physical intuition, the correlation part

of a fermionic wavefunction as $e^{-u\left(\left|\mathbf{r}_{i}-\mathbf{r}_{j}\right|\right)}$, i.e. a repulsive pseudopotential. Moreover, Wigner and Seitz [14], and Wigner [13], conclude that the conditional probability part contained into a fermionic wavefunction, must be of the form $f\left(\left|\mathbf{r}_{i}-\mathbf{r}_{j}\right|\right)$. This suggest that indeed, the physical principle we have used, that is a high Coulomb interaction corresponds to a less favorable situation compared to the situation of a weaker interaction, is a justified argument. Moreover, the exponential form given to the conditional probability, which resembles the behavior just explained and fulfills all the mathematical requirements, although not exact, cannot be far from the exact one , and thus, at least qualitatively, also the functional obtained is reasonably rigorous. A practical advantage of this approach is that it is not difficult, in principle, to implement this idea in a self-consistent calculation for atoms with a statitically relevant number of electrons (e.g. metals), where the Thomas-Fermi and Weiszacker terms describes well the the local part of the kinetic energy. In this case one

can use the spherical symmetry and then compare the results with those obtained using, for example, the Hartree-Fock method. This test will certainly show possible advantages or disadvantages of this approach and thus inspire new ideas to improve it.

\section{Acknowledgments}

We thank M.Deserno, R.A.Mosna and I.P.Hamilton for helpful comments.

[1] L.Delle Site,J.Phys.A 38, 7893 (2005).

[2] E.V. Ludeña, V.V.Karasiev and P.Nieto, Theor.Chem.Acc.110, 395 (2003).

[3] E.V. Ludeña and V.Karasiev, Kinetic Energy Functionals: History, Challenges and Prospects, in: K.D. Sen (ed.) Reviews of Modern Quantum Chemistry. A Celebration of the Contributions of Robert G. Parr, World Scientific, New Jersey, 612, (2002). 
[4] M.Kohout, Int.J.Quant.Chem. 87, 12 (2002).

[5] J.L.Gasquez and E.V. Ludeña, Chem.Phys.Lett. 83, 145 (1981).

[6] S.Watson and E.Carter, Comp.Phys.Comm. 128, 67 (2000).

[7] W.Yang and R.G.Parr, Density Functional Theory of Atoms and Molecules, Oxford University Press, New York, 1989.

[8] P.W.Ayers, J.Math.Phys. 46, 062107 (2005).

[9] R.A.Fisher, Proc.Cambridge Philoso.Soc. 22, 700 (1925).

[10] S.B.Sears, R.G.Parr and U.Dinur, Isr.J.Chem. 19, 165 (1980).

[11] A.Nagy, J.Chem.Phys. 119, 9401 (2003).

[12] E.Romera and J.S.Dehesa, J.Chem.Phys. 120, 8906 (2004).

[13] E.Wigner, Phys.Rev. 46, 1002 (1934).

[14] E.Wigner and F.Seitz, Phys.Rev. 46, 509 (1934).

[15] S.K.Gosh and B.M.Deb, Phys.Rep.92, 1 (1982).

[16] A Primer in Density Functional Theory, C.Fiolhais, F.Nogueira and M.Marques (Eds.), Lecture Notes in Physics, 620, Springer-Verlag, Berlin Heidelberg 2003.

[17] D.Ceperly, Phys.Rev.B 18, 3126 (1978).

[18] R.Jastrow, Phys.Rev. 98, 1479 (1955). 